\title{
MALDI In-Source Decay, from Sequencing to Imaging
}

\author{
Delphine Debois, Nicolas Smargiasso, Kevin Demeure, Daiki Asakawa, \\ Tyler A. Zimmerman, Loïc Quinton, and Edwin De Pauw
}

\begin{abstract}
Matrix-assisted laser desorption/ionization (MALDI) is now a mature method allowing the identification and, more challenging, the quantification of biopolymers (proteins, nucleic acids, glycans, etc). MALDI spectra show mostly intact singly charged ions. To obtain fragments, the activation of singly charged precursors is necessary, but not efficient above $3.5 \mathrm{kDa}$, thus making MALDI MS/MS difficult for large species. In-source decay (ISD) is a prompt fragmentation reaction that can be induced thermally or by radicals. As fragments are formed in the source, precursor ions cannot be selected; however, the technique is not limited by the mass of the analyzed compounds and pseudo MS3 can be performed on intense fragments. The discovery of new matrices that enhance the ISD yield, combined with the high sensitivity of MALDI mass spectrometers, and software development, opens new perspectives. We first review the mechanisms involved in the ISD processes, then discuss ISD applications like top-down sequencing and post-translational modifications (PTMs) studies, and finally review MALDI-ISD tissue imaging applications.
\end{abstract}

Keywords Imaging • In-source decay $\bullet$ MALDI • Post-translational modifications - Sequencing

\section{Contents}

1 Introduction

2 ISD Mechanisms

2.1 Radical-Induced Pathway

2.2 Collisionally Activated Pathway

D.Debois, N.Smargiasso, K.Demeure, D.Asakawa, T.A.Zimmerman, L.Quinton and E.De Pauw $(\square)$

Mass Spectrometry Laboratory, GIGA-R, Department of Chemistry

University of Liège, Allée de la Chimie 3, 4000 Liège, Belgium

e-mail: e.depauw@ulg.ac.be 
2.3 Influence of the Matrix

2.4 Radical-Induced Pathway via Hydrogen Abstraction

3 Proteomic Applications of MALDI-ISD

3.1 MALDI-ISD and Bottom-Up Strategies

3.2 MALDI-ISD and Top-Down Strategies

3.3 MALDI-ISD and Pseudo-MS ${ }^{3}$ Strategies

3.4 MALDI-ISD and PTMs

3.5 MALDI-ISD of Oligonucleotides

4 MALDI Mass Spectrometry Imaging

4.1 MALDI-ISD on Tissue Slices

4.2 MALDI-ISD Imaging

5 Conclusions

References

\section{Abbreviations}

$\begin{array}{ll}\text { 2-AA } & \text { Anthranilic acid } \\ \text { 2-AB } & \text { 2-Aminobenzamide } \\ \text { 5-ASA } & \text { 5-Aminosalicylic acid } \\ \text { ATT } & \text { 6-Aza-2-thiothymine } \\ \text { BLAST } & \text { Basic Local Alignment Search Tool } \\ \text { CHCA } & \alpha \text {-Cyanohydroxycinnamic acid } \\ \text { CID } & \text { Collision induced dissociation } \\ \text { 1,5-DAN } & \text { 1,5-Diaminonaphthalene } \\ \text { 2,5-DHB } & \text { 2,5-Dihydroxybenzoic acid } \\ \text { ECD } & \text { Electron capture dissociation } \\ \text { ED } & \text { Edman degradation } \\ \text { ETD } & \text { Electron transfer dissociation } \\ \text { (HP)LC } & \text { High performance liquid chromatography } \\ \text { ISD } & \text { In-source decay } \\ \text { MALDI } & \text { Matrix-assisted laser desorption/ionization } \\ \text { MS/MS } & \text { Tandem mass spectrometry } \\ \text { PMF } & \text { Peptide-mass fingerprinting } \\ \text { PSD } & \text { Post-source decay } \\ \text { PTM } & \text { Post-translational modification } \\ \text { TDS } & \text { Top-down sequencing }\end{array}$

\section{Introduction}

The field of biopolymer analysis has evolved considerably over the past 10 years [1]. A major example is the explosion of "omics" techniques. Mass spectrometry has had a major impact resulting from the continuous development of MS-based methodologies, including instrumentation and comprehensive strategies for the identification and quantification of a large number of molecules during a single experiment [2-4]. The next challenge is their localization in biological samples with their simultaneous identification and quantification. 
Matrix-assisted laser desorption/ionization (MALDI) played a large part in these developments [5]. In UV-MALDI, the energy of a laser pulse is dissipated by a UV-absorbing matrix containing the analytes and a dense gas phase is produced (plume) from which intact ions survive. The primary mechanisms are not yet fully understood but probably consist of mixed processes involving photo-excitation, collision-induced desolvation of clusters, and gas phase acid-base chemistry leading to charge transfer. The role of the MALDI matrix is crucial to enhance the ion signal, and besides "general purpose" matrices, specific matrices are adapted for various families of analytes [6]. Matrices have been classified as hot or cold according to the internal energy transferred to the analytes, and many papers describe methods to study the energetics and kinetics of the MALDI process [7]. Among important processes, gas phase reactions can increase the internal energy of ions by their exothermicity. In contrast, multiple gas phase collisions have a cooling effect [8]. The balance between these processes will govern the final energy state of the ion population, and therefore the fragmentation rate constants and the resulting mass spectra. If fragmentation occurs in the source, the ions will be detected at their expected $\mathrm{m} / \mathrm{z}$ values. The decay of this population of ions is called in-source decay (ISD). When the fragmentation reaction is too slow to occur in the source but fast enough to occur before detection, the ions are called metastable and the decay of their population is called post-source decay (PSD) [9]. Dedicated methods of detection are required [10].

Two major strategies rely on mass spectrometry in biopolymer analysis - the classical bottom-up approach and the top-down strategy. In the "bottom-up" approach, biopolymers are first digested into oligomers. In the case of proteins, the sample preparation consists of the reduction of the disulfide bridges, followed by alkylation of the sulfhydryl groups and an enzymatic digestion. The resulting mix of peptides is then analyzed by MALDI. If the sample is complex, a 2D gel separation is performed. This method is known as PMF for "peptide-mass fingerprinting". It can be followed by MS/MS for sequencing the peptides using PSD to increase the identification score. Another mass spectrometric approach commonly employed to sequence proteins is "top-down" sequencing (TDS) [11]. This strategy generally relies on the direct fragmentation of the intact protein in the gas-phase, i.e., without any enzymatic digestion before mass spectrometry. The fact that the whole sequence is accessible to fragmentation is one of its major advantages. TDS is usually performed on multiply charged ions generated from electrospray ion sources. Fragmentations occur by collision induced dissociation (CID), by electrons as in electron capture dissociation (ECD) $[6,12,13]$, or by electron transfer dissociation (ETD) [14]. Until recently, TDS was not intensively used with MALDI due to the fact that the produced ions are singly charged and MS/MS techniques available on MALDI mass spectrometers are efficient only for peptides up to $\mathrm{m} / z$ 3,500 [12]. Indeed, PSD and CID fragmentation processes rely on a slow increase of the ion internal energy by multiple collisions with gas molecules until the lower energy bonds are dissociated (mainly labile or peptide bonds). In addition, for higher masses, the energy deposited is not sufficient to induce dissociation. However, as the ion formation in ISD occurs before the extraction, fragments even from large proteins receive the full kinetic energy, 
fly at their own velocity, and are detected directly at the true $m / z$ value. The abundance of these fragments is usually low and ISD is generally seen as an unwanted side process and is therefore minimized. The ISD fragment nature depends on the MALDI matrix chosen and the chemistry leading to the fragmentation.

In this chapter we will show that ISD can be a powerful structural analysis tool when promoted by specific MALDI matrices presenting high ISD yield. The following review is divided into three parts:

1. The description of the mechanisms leading to ISD fragmentation during the MALDI process. We will describe the two main mechanisms of ISD fragmentation and the influence of the matrix used.

2. The description of typical MALDI-ISD spectra obtained with peptides and proteins. We will demonstrate the interest in using MALDI-ISD to sequence proteins as well as the limitations of this approach and the possible solutions. We will also review the application of ISD to biopolymers.

3. The application of MALDI-ISD to tissue imaging. We describe a new application of ISD to mass spectrometry imaging (MSI). We demonstrate that ISD can be performed directly on a tissue slice and that the correlation between a fragment and its localization leads to identification of different protein isoforms.

\section{ISD Mechanisms}

Ions produced by soft ionization methods like MALDI are generally closed valence shell ions, which mostly undergo rearrangement type fragmentations. These reactions are often characterized by a low activation enthalpy and a negative activation entropy. Under low energy activation conditions, they give access to low energy fragmentation channels. Fast thermal activation can be achieved when sufficient energy is transferred from the matrix (hard matrices) but fast reactions have also been shown to occur through radical chemistry. Recently, new matrices able to release hydrogen radicals upon laser irradiation have been developed showing an increase in the yield of ISD fragments [15].

Mechanistically, ISD is not yet fully understood. In MALDI, the prompt in-source fragmentation can have two origins - thermal or chemical activation. Once the laser energy is dissipated into the matrix, a "plume" containing intact ions is produced [6]. Several phenomena can then occur, such as the fragmentation of matrix clusters and gas phase acid-base chemistry (proton transfer). Different matrices have been classified as "hot" or "cold" according to the internal energy transferred to the analytes. Chemical activation by hydrogen radicals is another mechanism inducing a prompt "in source" fragmentation reaction [16, 17]. The matrix releases hydrogen radicals. Radicals can react with the analyte to produce radical species that will undergo specific fragmentation reactions. Using radical scavengers, it has been shown that the ability of matrices to release hydrogen radicals is directly linked to the efficiency of ISD fragmentation. The mass accuracy and resolution of reflectron 
TOF instruments allow the unambiguous observation of the isotopic distribution of these fragments and clearly shows reduction reactions to occur.

The balance between thermal and radical activation will govern the chemical state (closed shell or radical) and the internal energy of the ion population, and therefore the fragmentation channels and their respective rate constants. Interestingly, these radical species are generated and induce fragmentations independently from the mass of the analytes. In the case of proteins, large $c$-ion and $z$-ion series detected up to $\mathrm{m} / \mathrm{z}$ 10,000 allow for fast and efficient sequencing of the $\mathrm{N}$ - and $\mathrm{C}$-termini extremities. The range $0<m / z<800$ is not accessible as intense matrix signals hide both $\mathrm{C}$-terminal and $\mathrm{N}$-terminal ISD fragments. This limitation can be overcome by the use of pseudo-MS ${ }^{3}$ strategies.

A study on the internal energy build-up of benzylpyridinium ions formed in MALDI revealed that fragmentation can be due to thermal activation, and the effective temperatures were estimated [17]. It has been proposed that the exothermicity of acid-base reactions can lead to an internal energy increase [6]. The ion's initial velocity is directly proportional to the rate of dissipation of the plume $[13,17]$. Thus, matrices characterized by a low exothermicity of acid-base reaction and by a high initial velocity are usually colder because the analytes have less probability of interacting with the rest of the plume.

ISD-suitable MALDI matrices revived interest in using the ISD for the de novo sequencing of peptides and proteins [18, 19]. Indeed, ISD experiments can be realized on all MALDI-TOF mass spectrometers, widely available in mass spectrometry laboratories and in proteomics facilities, just by using a suitable matrix. Sakakura et al. recently used the 5-aminosalicylic acid matrix (5-ASA) and demonstrated that this matrix is more efficient than the classical 2,5-DHB to produce $c$ - and $z$-fragments [20]. However, this matrix remains less efficient than the 1,5-DAN matrix and rather difficult to use (low solubility) but possesses the advantage of being non-carcinogenic. Very recently, two additional molecules were shown to induce radical-induced fragmentation of peptides and proteins - 2-aminobenzoic acid (2-AA, also known as anthranilic acid) and 2-aminobenzamide (2-AB) [21].

The 1,5-DAN matrix mainly leads to (1) a radical mediated fragmentation pathway, leading to the formation of $c$ - and $z$-fragments that can be fully annotated $\left(\mathrm{C}^{\prime}\right.$ and $\left.\mathrm{Z}^{\prime}\right)$ and (2) a thermal activation induced by the laser irradiation, and mediated by the matrix which leads mainly to CID-like $y-, a$-, and $b$-fragments. In some cases, the very useful $d$ - and $w$-type fragments are also observed [15]. These latter imply a side-chain loss whose mass depends on the amino acid residue and can therefore be useful to confirm a residue determined by two consecutive $c$ - or $z$-fragments or even to distinguish the isobaric amino acid residues leucine and isoleucine [22]. Bache et al. have demonstrated that ISD fragmentation occurs with a low level of hydrogen scrambling, conserving the solution deuteration pattern in the gas phase fragments formed. This fragmentation technique could therefore be applied to hydrogen/ deuterium exchange studies of peptides and small proteins [23, 24]. 


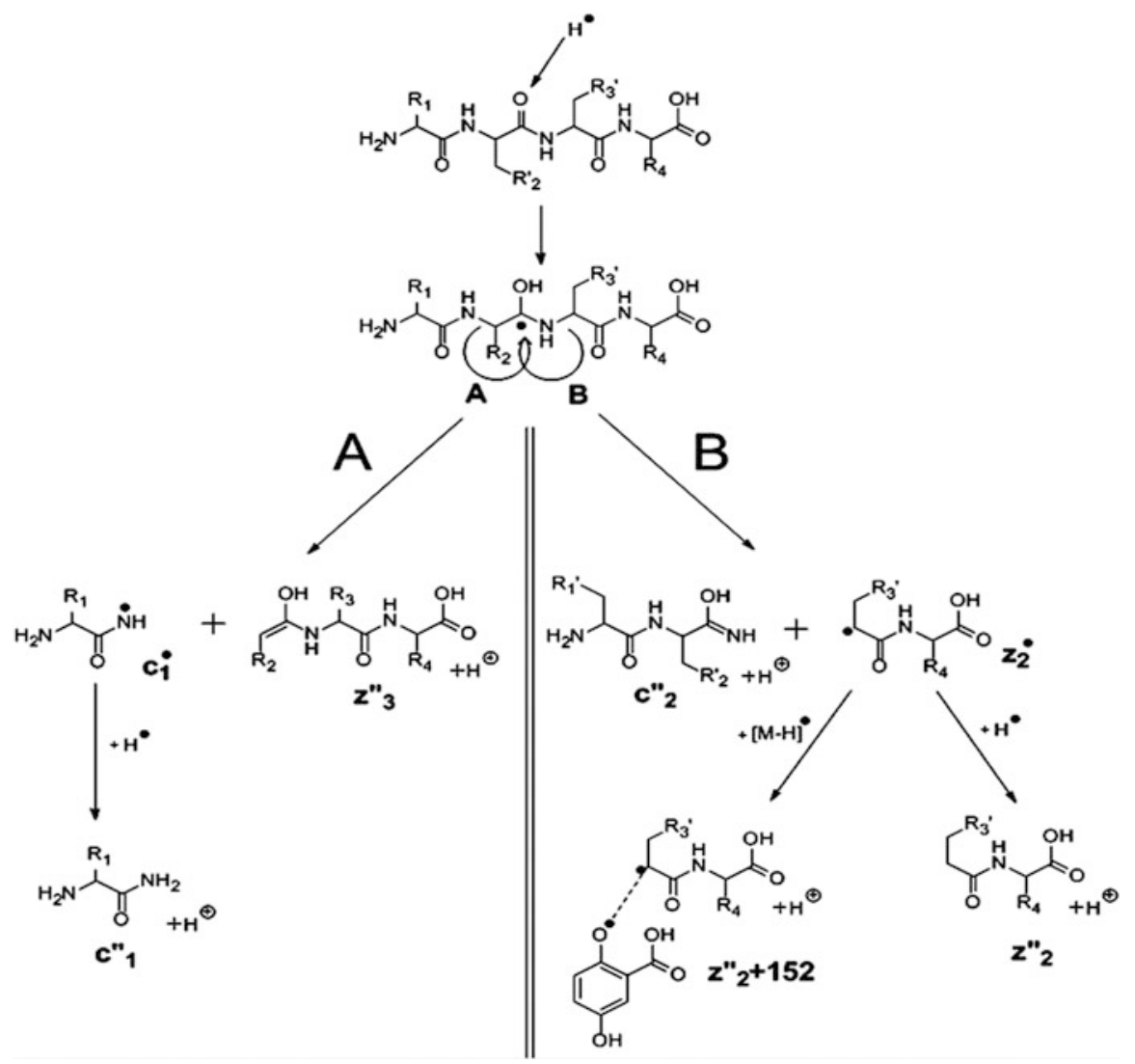

Scheme 1 Mechanism of the formation of radical-induced $c$ - and $z$-fragments

\subsection{Radical-Induced Pathway}

Hydrogen radicals formed by the matrix are at the origin of the formation of the $c$ - and $z$-fragments [25-27], as depicted in a mechanism proposed by Takayama [25]. In the matrix crystals, hydrogen bonds are formed between the matrix and the analytes. During UV irradiation, a hydrogen radical transfer occurs from the matrix to the peptide, leading to an unstable radical that is rapidly cleaved to give $c$ - and $z$-fragments (Scheme 1). $z$-fragments can also form adducts with a DHB molecule to give $[z+2]+152$ fragments using the 2,5-dihydroxybenzoic acid matrix. The authors also conclude that the photoelectrons are involved in the ISD fragmentation mechanism [27].

The radical-induced ISD efficiency of a matrix can be correlated to its tendency to transfer hydrogen radicals, and this ability can be tested with hydrogen scavengers [28, 29] such as spirooxazines [18]. The radical-induced ISD efficiency of a matrix can also be correlated to its tendency to reduce the disulfide bond of 
peptide [15] and this strategy was used by Sakakura et al. to classify 5-ASA amongst the other ISD matrices [20].

Based on these results, a ranking of the different matrices, from the least to the most able to transfer hydrogen radicals, was deduced: $\mathrm{CHCA} \ll 2,5$-DHB $<$ 2 -AA $<5$-ASA $\approx 2-\mathrm{AB}<1,5$-DAN. This ranking is in good agreement with the increasing order of their radical-induced ISD abilities. Another characteristic of radical-induced ISD is its independence from the ionization process [30]. Indeed, Takayama et al. suggest that basic amino acids near the $\mathrm{N}$-terminus favor production of $c$-type fragments and basic amino acids near the $\mathrm{C}$-terminus favor production of $z$ and $y$-type fragments in positive ion mode [31, 32]. As ISD is independent of the ionization process, radical-induced ISD fragments are also observed in the negative ion mode according to the ability of the matrix to produce negatively charged molecular ions. In negative mode, the 1,5-DAN matrix is far more efficient than the 2,5-DHB matrix [15, 18]. The radical-induced fragmentation mechanism is exactly the same in both ion modes [15].

\subsection{Collisionally Activated Pathway}

For the thermal pathway, the activation of the fragmentation process of peptides occurs by a direct energy transfer from the matrix to the peptide and by collisions with matrix molecules in the expanding MALDI plume formed following laser irradiation $[16,17]$. This thermal pathway leads to the CID-like $a$-, $b$ - and $y$-ions corresponding to their PSD analogs. PSD is the metastable fragmentation occurring outside the MALDI source. This fragmentation process is activated by bimolecular collisions with the plume components during the ion extraction. The PSD fragmentation leads to the formation of the CID-like $a$-, $b$ - and $y$-fragments.

\subsection{Influence of the Matrix}

The two kinds of ISD pathways are differently favored according to the matrix used. The in-source formation of CID-like fragments by the thermal pathway has not been widely studied [33]. Because this thermal process is caused by vibrational activation of closed valence shell ions, the fragmentation mechanism can be explained by the "mobile proton" model [34]. These fragments are the result of the cleavage of the $\mathrm{CO}-\mathrm{NH}$ bond that is weakened by the protonation of the nitrogen of the amide group [35, 36]. The CID-like fragments ( $a-, b$-, and $y$-type) can then be favored by the acidity of the matrix. The 2,5-DHB matrix is then more efficient than the 1,5-DAN matrix for production of these ions. However, it was shown by Sachon et al. with labile peptides that the formation of these fragment types cannot be explained only by the proton affinities of the matrices [37]. The low energy bimolecular collisions between the peptide and the matrix can activate 
the fragmentation and are mediated by the initial axial velocity of the ions of the MALDI plume. The lower abundance fragments issued from this pathway using the 1,5-DAN matrix can make ISD spectra easier to interpret. Nevertheless, use of the 1,5-DAN and 2,5-DHB matrices can be complementary to facilitate the interpretation of ISD spectra of unknown peptides or proteins. It is noted that the formation of CID-like fragments tends to disappear with the increasing molecular weight of the studied compound.

\subsection{Radical-Induced Pathway via Hydrogen Abstraction}

Recently it was found that the oxidizing matrices 5-formylsalicylic acid and 5-nitrosalicylic acid for MALDI-ISD resulted in the generation of $a$ and $x$ ions by cleavage of the $\mathrm{C}_{\alpha}-\mathrm{C}$ bond. MALDI-ISD with an oxidizing matrix is initiated by hydrogen abstraction from amide portion of the peptide backbone onto the matrix, as depicted in a mechanism proposed by Asakawa and Takayama [38, 39]. Hydrogen abstraction from peptides resulted in the formation of oxidized peptide molecules $[\mathrm{M}-\mathrm{H}]^{\bullet}$ containing a radical site on the amide nitrogen. Subsequently, the $[\mathrm{M}-\mathrm{H}]^{\circ}$ radical principally generated the $a$ and $x$ ions, indicating cleavage of the $\mathrm{C}_{\alpha}-\mathrm{C}$ bonds on the peptide backbone. However, the $x$ ions originating from the cleavage of the $\mathrm{C}_{\alpha}-\mathrm{C}$ bonds at $\mathrm{Xxx}$-Pro sequences were absent, because the Pro residue cannot have a nitrogen-centered radical site. The $\mathrm{C}_{\alpha}-\mathrm{C}$ bond cleavage at $\mathrm{Xxx}$-Pro and Pro-Xxx bonds would lead to $a / x$. and $a / x$ fragment pairs, respectively. The absence of $x$ ions originating from the cleavage of the $\mathrm{C}_{\alpha}-\mathrm{C}$ bonds at Xxx-Pro indicates that the fragmentation leading to an $a / x \cdot$ ion pair does not occur. However, the radical fragments $a \cdot$ ions were not observed in MALDI-ISD spectra with oxidizing matrix, and instead the $a$ ions were detected. It is likely that there are sufficient amounts of exited matrix molecules and matrix radicals in the MALDI plume to form the $a$ ions via further hydrogen abstraction after the $\mathrm{C}_{\alpha}-\mathrm{C}$ bond cleavage. The fragmentation mechanism in MALDI-ISD with oxidizing matrix is shown in Scheme 2a. In contrast, the cleavage of the $\mathrm{C}_{\alpha}-\mathrm{C}$ bond at $\mathrm{Xxx}-$ Pro did not occur, and instead $\mathrm{CO}-\mathrm{N}$ bond cleavage at $\mathrm{Xxx}-\mathrm{Pro}$ was observed via hydrogen abstraction from the $\mathrm{C}_{\alpha}-\mathrm{H}$ bond in the Pro residue. The CO-N bond cleavage leads to the formation of the $b / y$ fragment pair and the $b$-ions undergo further degradation to form the $b$ and $a$ ions after the CO-N bond cleavage, as shown in Scheme 2b [39].

\section{Proteomic Applications of MALDI-ISD}

\subsection{MALDI-ISD and Bottom-Up Strategies}

As MALDI-ISD occurs in the source, this fragmentation method does not allow any precursor selection. In consequence, when working on enzymatic digests ISD 
<smiles>[R]C=NC(=O)C([R])N(C)CCN(CC)C([R])C(=O)N(CC)CC</smiles>

b<smiles>[R]C(C(=O)N1CCN(C)C1)N(C)C</smiles><smiles>[R]C(C=O)N(C)CC</smiles><smiles>CC1=NCCC1</smiles><smiles>[R]C=NC(=O)OCCCCCCC</smiles>

Scheme 2 Mechanism of MALDI-ISD with oxidizing matrix. (a) $\mathrm{C}_{\alpha}-\mathrm{C}$ bond cleavage and (b) $\mathrm{CO}-\mathrm{N}$ bond cleavage at $\mathrm{Xxx}-$ Pro bond

signals from peptides will co-occur and the resulting overlapping spectra will be difficult to untangle. A purification step and separation of the peptides by LC-MALDI is thus necessary to overcome this limitation. The presence of matrix clusters in the low $\mathrm{m} / \mathrm{z}$ range $(<700 \mathrm{Da})$ may also burden the observation of low-mass fragments. Information cannot be easily obtained from this mass range, reducing the experimental observation window. However, Reiber and Brown have shown the possibility to sequence de novo purified small peptides in spite of matrix adducts in the spectral low mass range [40]. The authors combined the information coming from $\mathrm{N}$-terminus ( $c$-ions) and $\mathrm{C}$-terminus ( $y$-ions) fragments to identify six unknown peptides. Even if the authors underlined several ambiguities in their sequences, this approach highlights that two types of fragments can be used to bypass the loss of information caused by the low mass matrix clusters. Another methodology for de novo sequencing of peptides using MALDI-ISD has been recently demonstrated by Quinton et al. [19]. The authors have used PSD and ISD spectra in combination to determine sequences which were indecipherable by a single fragmentation method. 


\subsection{MALDI-ISD and Top-Down Strategies}

MALDI-ISD appears to be a good answer to perform TDS because, as explained above, the mechanism partially relies on a transfer of radical species and the fragmentation process is consequently not limited by the mass. Thus, different groups tried to sequence purified proteins spotted in sinapinic acid (SA) or 2,5-DHB. In 1995, MALDI-ISD was performed on different proteins such as bovine cytochrome $c(12.2 \mathrm{kDa})$, bovine superoxide dismutase $(15.6 \mathrm{kDa})$, and equine apomyoglobin $(16.9 \mathrm{kDa})[41,42]$. Numerous intense $c-, z-$, and $y$ - fragments were observed between $850 \mathrm{~m} / \mathrm{z}$ and $11,100 \mathrm{~m} / \mathrm{z}$. Figure 1 displays two MALDIISD spectra acquired for horse heart cytochrome $c(12.2 \mathrm{kDa})$ and myoglobin $(16.9 \mathrm{kDa})$. Clear $c^{-}, z_{-}$, and $y$-ion series are detected and lead to the characterization of 78 amino acids out of 104 for cytochrome $c(75 \%)$ and 93 out of 153 for myoglobin $(61 \%)$.

These signals enable the building of long sequence tags for each protein, which can be submitted in BLAST in order to compare them to the sequences present in the databases and to identify the protein of interest. However, the full sequence of the protein is not always accessible by ISD. For example, as the ISD process leads to breaking the $\mathrm{N}-\mathrm{C}_{\alpha}$ bond, a proline residue cannot produce ISD fragments due to its cyclic nature. However, this observation is predictable, and a gap in $c$-ions of an ISD-sequencing experiment can be interpreted as a proline residue on the $\mathrm{N}$-side followed by another amino acid, Pro- $\mathrm{X}$, the mass and position of which in the sequence can easily be determined.

\subsection{MALDI-ISD and Pseudo-MS ${ }^{3}$ Strategies}

Protein ISD generally leads to an intense $c$-ion series and allows a precise characterization of the N-terminus part of the protein(s). However, the full $\mathrm{N}$ - and C-termini cannot be determined due to matrix background at low $\mathrm{m} / \mathrm{z}$ ratios that prevents the observation of the very first fragments. Different "pseudo-MS $\mathrm{M}^{3}$ " $\left(\mathrm{pMS}^{3}\right)$ strategies have therefore been imagined to describe the sequences extremities $[43,44]$. These strategies exploit the fact that an ISD fragment can be selected and fragmented by CID like a classical ion generated by a MALDI source. Simply, an ISD fragment $(m / z<3,500)$ is isolated before subjection to PSD fragmentation. If the selected ion is a $c$-type, then the resulting MS/MS spectrum will characterize the $\mathrm{N}$-terminus. Similarly, if the precursor ion is a $z$ - or a $y$-ion, then the C-terminus part will be characterized. Figure 2 shows an example of a $\mathrm{pMS}^{3}$-experiment. Aldolase protein $(39.2 \mathrm{kDa})$ has been fragmented by MALDI-ISD using 2,5-DHB. A tag of 56 consecutive amino acids has been obtained from this single spectrum. However, as already discussed above, the $\mathrm{N}$ - and the $\mathrm{C}$-termini are missing. To characterize the C-terminus part, a $y$-ion $\left(y_{12}\right)$ was then selected and fragmented. The MS/MS spectrum obtained (Fig. 2, 

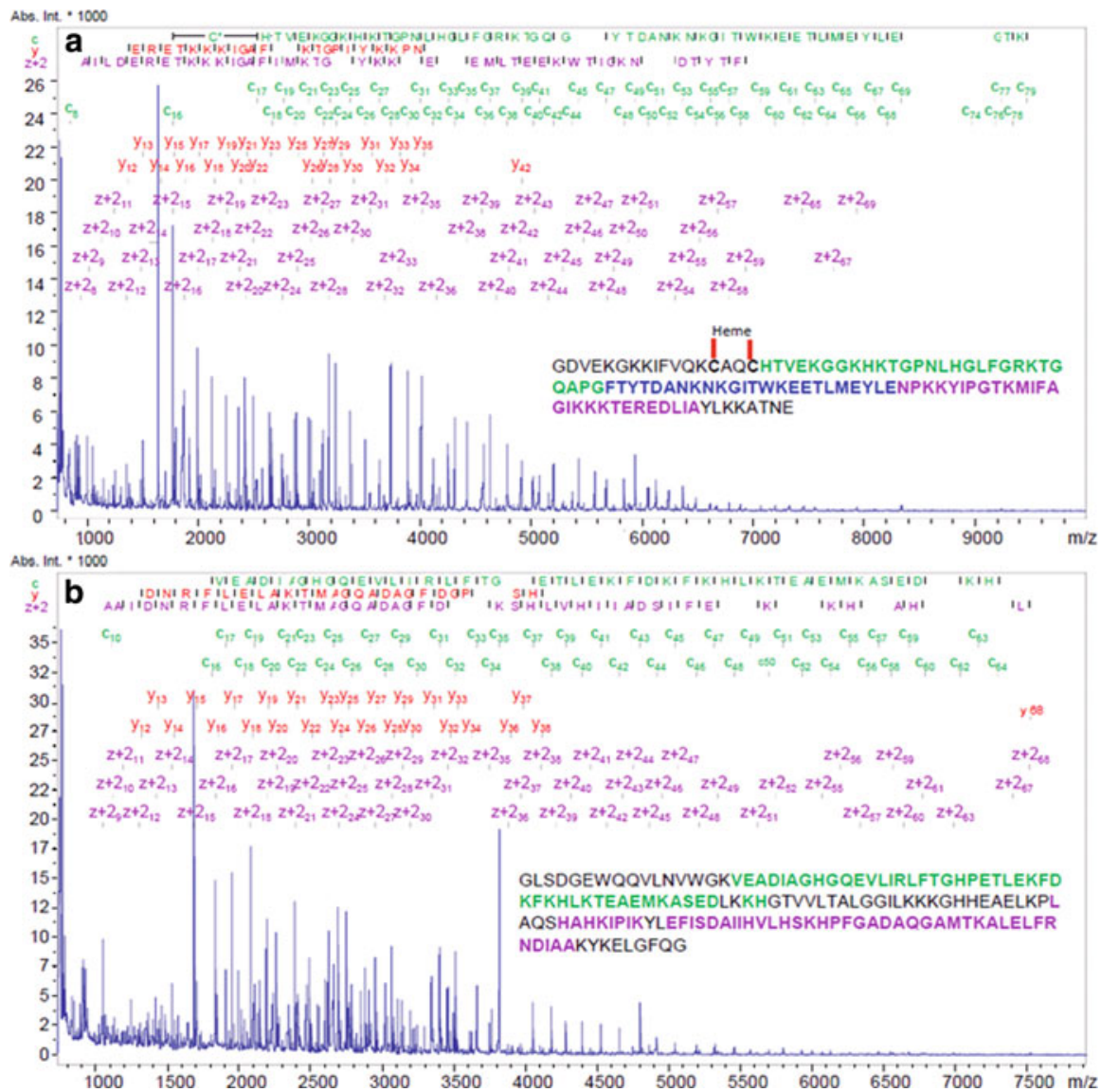

Fig. 1 MALDI-ISD spectra of (a) horse heart cytochrome $c(12.2 \mathrm{kDa})$ and (b) horse heart myoglobin $(16.9 \mathrm{kDa})$. One picomole of each protein was spotted onto the MALDI plate and mixed together with 1,5-DAN saturated in FA $(0.2 \%) / \mathrm{ACN}$. Using intense $c$ - and $z$-ion series, 77\% and $61 \%$ of sequence coverage were characterized for cytochrome $c$ and myoglobin, respectively. $\mathrm{C}$ - and $\mathrm{N}$-terminus extremities are missing because fragments are hidden by intense matrix signals

bottom panel) clearly displays intense $y$ - and $b$-ion types which enable an easy characterization of the missing C-terminus part.

As the use of $\mathrm{pMS}^{3}$-sequencing allows full characterization of the protein extremities, it constitutes a competitive methodology to replace Edman degradation (ED). ED was the most used protein sequencer during the past few decades. First of all, the time scale of each experiment is in favor of ISD/pMS ${ }^{3}$. Whereas ED takes more than $30 \mathrm{~min}$ per amino acid, MALDI-ISD takes around $10 \mathrm{~min}$ from the sample deposition to determination of a tag of several tens of amino acids. Moreover, from tens of picomoles of purified proteins, ED allows the sequencing of the first 50 amino acids except when the $\mathrm{N}$-terminal amino acid is modified. With 


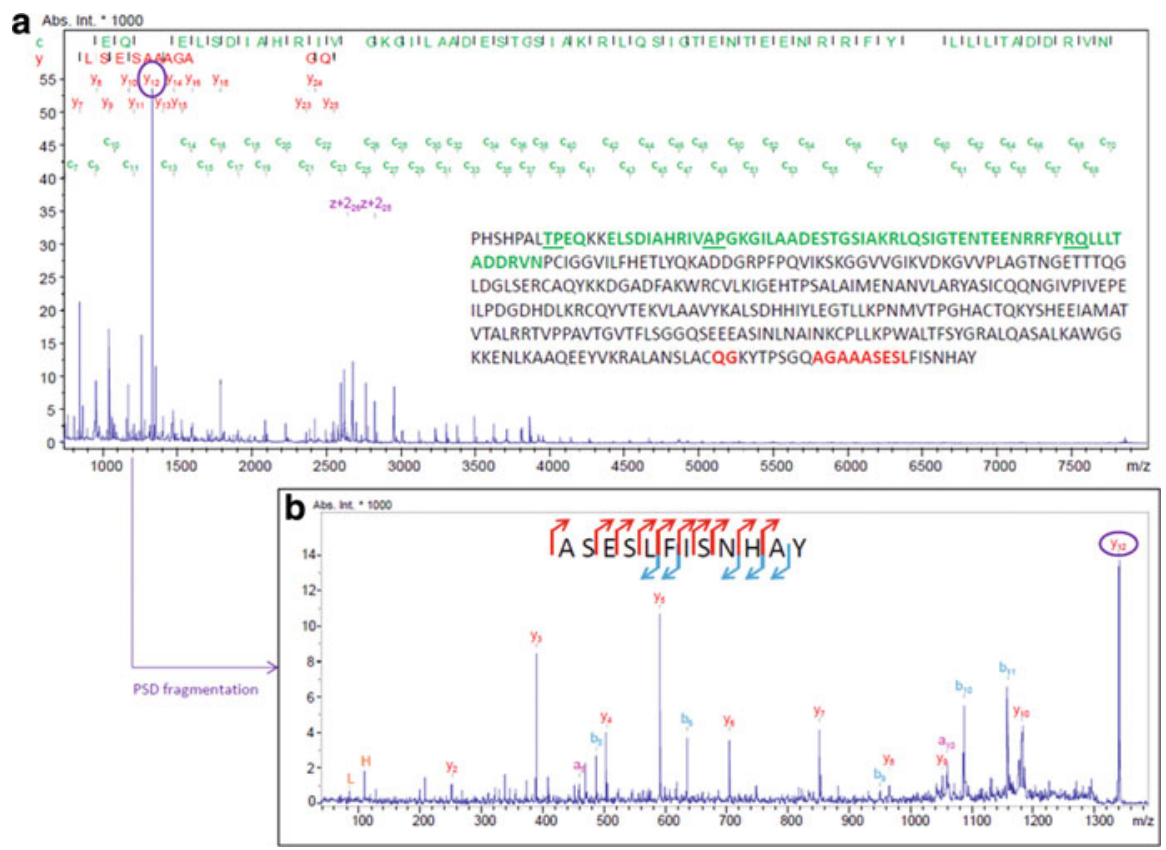

Fig. 2 Example of a $\mathrm{pMS}^{3}$ experiment on Aldolase (39.2 kDa). (a) MALDI-ISD spectra of the protein displaying a long series of $c$-ions but also several $y$ - and $z$-type ions. (b) $y_{12}$ ion is fragmented by post-source decay, unambiguously revealing the whole missing $\mathrm{N}$-terminus extremity

the same amount of compound or even less, MALDI-ISD combined with $\mathrm{pMS}^{3}$-sequencing gives access in the more favorable cases to the first 80 amino acids from the $\mathrm{N}$-terminus but also to the last 40 amino acids from the $\mathrm{C}$-terminus.

It is important to highlight that $\mathrm{pMS}^{3}$-sequencing also works if the extremities are modified and, thus, can reveal post-translational modifications (PTMs). The approach of combining ISD and $\mathrm{pMS}^{3}$ has recently been highlighted by Resemann et al. [45]. In this work, the authors demonstrated the ability of MALDI-TDS to sequence fully a Camelid single heavy chain antibody of $13.6 \mathrm{kDa}$. The authors indicate that larger proteins may also be sequenced by MALDI-TDS but the use of reference sequence information in databases or complementary results from bottom-up approaches becomes unavoidable.

\subsection{MALDI-ISD and PTMs}

Several teams tried to apply MALDI-ISD to the detection and localization of PTMs. In 1997, Lennon and Walsh demonstrated that sequence determination by MALDI-ISD was stopped near the cysteins involved in disulfide bonds or covalent bonds to a heme molecule. These results demonstrated that the peptide 
modifications were able to interfere with the ISD promoted by DHB [42, 44]. Indeed, a disulfide bond tends to be reduced during the ISD process [46] and therefore acts as a quencher of the fragmentation of neighboring amino acids. This observation was exploited by Schnaible et al. to localize disulfide bonds on HPLC-separated peptides [47, 48]. Moreover, PTMs that do not interfere with the ISD fragmentation of the peptide backbone can be studied by ISD as well. For example, Lennon et al. demonstrated in 1999 that labile modifications such as serine phosphorylations were not affected by the ISD process [49]. These PTMs can therefore be characterized either by a direct MALDI-ISD experiment or after a $\mathrm{pMS}^{3}$-sequencing methodology [50].

Concerning glycosylation, MALDI-ISD was applied both on glycoproteins/ glycopeptides and on isolated glycans. Collisionally-induced MALDI-ISD of glycopeptides led to the observation of both glycan and peptide fragmentations [51]. On the other hand, radical-induced ISD allowed Hanisch sequencing glycoproteins and localizing O-glycosylation sites, confirming again the ability of this technique to preserve labile PTMs [52]. However, Chaurand et al. observed that $\mathrm{N}$-glycosylation was able to stop the protein sequencing, allowing localization of this PTM, but not further sequencing [53]. These contradictory results suggest a potential effect of the carbohydrate moiety on the sequencing of the glycoproteins.

As glycans can be easily isolated and purified, the ISD and $\mathrm{pMS}^{3}$-sequencing approaches were also employed to characterize thoroughly the structures of both native [43, 54-60] and permethylated glycans [61-66], allowing unambiguous discrimination of isobaric species. In ref. [64] it was also mentioned that 6-aza-2thiothymine (ATT) was able to promote ISD fragmentation of permethylated glycans. The mechanism involves in this case the protonation of the permethylated glycan, leading to its fragmentation. In addition, 2,5-DHB could act as a dual matrix here, allowing ISD of permethylated glycans when laser shots are made on crystals (where protons are available) but impeding ISD when laser shots are made in the central amorphous zone of the spots. Indeed, this part is sodium-rich and protonation is therefore unfavored compared to cationization [67].

Other classical PTMs such as pyroglutamic acid [53], formylation [68], deamidation [44], and acetylation [2] have also been described and studied by MALDI-ISD. Finally, Yoo and collaborators showed recently that MALDI-ISD was also an appropriate tool to localize PEGylation of protein [69], which is a chemical modification widely employed in the pharmaceutical field [70].

In conclusion, MALDI-ISD can be employed in a wide panel of applications such as the characterization of recombinant proteins, the full de novo sequencing of unknown proteins, and the localization of PTMs.

\subsection{MALDI-ISD of Oligonucleotides}

Besides peptides and proteins, substantial work was also achieved in order to use ISD to sequence oligonucleotides. In 1995, Nordhoff et al. reported the prompt fragmentation of oligonucleotides in an IR-MALDI source [71] while Zhu et al. used 2,5-DHB to induce in-source fragmentation in a UV-MALDI source [71, 72]. 
Following these observations, delayed extraction was applied and sequencing of an 11-mer DNA was achieved using a $266 \mathrm{~nm}$ laser with picolinic acid as matrix [73]. According to the McLuckey nomenclature [74], mainly $w$-type ions (resulting from the cleavage of a phosphodiester bond and containing a $5^{\prime}$-terminal phosphate group) were observed. A base loss is considered to be a critical step of this "thermal" fragmentation pathway [75, 76]. Alternatively, a pathway of electronic energy transfer was also proposed to occur in positive ion mode with 2-aminobenzoic acid and leads to the formation of supplementary ions ( $d-, b$-, and $y$-ions) [77]. Concerning applications of ISD in the nucleic acids field, ISD was successfully applied by Wang et al. to sequence covalently modified (phosphorothioate instead of phosphodiester bonds) oligonucleotides [78] but failed to allow locating covalent rhodium adducts on DNA [79].

\section{MALDI Mass Spectrometry Imaging}

MALDI-MSI is a powerful technique that allows mapping of many classes of compounds directly from a tissue slice of several micrometers thickness. The principle of MALDI-MSI relies on a pixelization of the surface to be analyzed. A mass spectrum is recorded at each pixel, providing a triplet of information (spatial coordinates, mass, and intensity of the peak) for each signal present in the mass spectrum. Accumulating and integrating these data allows building a 2D cartography for any detected molecule, without the need for previous knowledge of the molecular content of the sample. On the image, the intensity of the signal is depicted by a color scale.

MALDI-MSI is suitable for high molecular weight biomolecules (proteins) but also for small molecules such as lipids, drugs, and their metabolites, etc. In most published applications, proteins are targeted as potential biomarkers of pathology, as in many studies, for ovarian tumors [80, 81], renal carcinoma [82], or Parkinson's disease [83, 84]. For small molecules analysis, two main classes may be distinguished - exogenous drugs and endogenous metabolites. Exogenous drugs have been studied in MALDI-MSI far later than proteins (since 2004) for different targets such as olanzapine (antipsychotic) [85-87], imatinib [87], vinblastine [88], and banoxantrone [89] (cancer drugs). Among endogenous metabolites, lipids such as glycerophospholipids and glycosphingolipids or simple lipids like cholesterol, diacylglycerols, or triacylglycerols have been extensively investigated [90]. Also studied are primary metabolites (ADP, ATP, . .) as targets for MALDI-MSI [91].

Concerning MALDI-MSI, a lot of work has been done to improve the experimental workflow, such as optimization of sample preparation and matrix deposition [92], and much focus has been on the identification of the detected molecules. When small molecules are analyzed, the identification is rather straightforward where tandem mass spectrometry can be easily used, leading to the fragmentation of the detected compounds, allowing their unambiguous 
characterization. The identification is even more reliable when high resolution exact mass measurements can be performed. If proteins are analyzed, it is rather difficult to perform MS/MS analysis directly from proteins that are more difficult to fragment and the unique information of the $\mathrm{m} / \mathrm{z}$ value is not sufficient as it may correspond to several primary sequences. To achieve protein identification, in situ digestion has been used since 2007 [93]. This method consists of the deposition (more often by micro-spotting or nano-spotting) of a solution of protease (usually trypsin) at the surface of the sample, followed by $2-4 \mathrm{~h}$ of incubation and analysis of the resulting mix of peptides. The classical PMF method is then used to query protein databases for identification. Although this procedure presents some drawbacks (need for a microspotting system to deposit the enzyme solution as precisely as possible, time-consuming, and leads to mass spectra which can be complex and difficult to interpret), it has been extensively used since its implementation. MALDI-ISD, however, removes the need for in situ digestion when analyzing tissues.

\subsection{MALDI-ISD on Tissue Slices}

MALDI-ISD seems to be useful in this context, and Debois et al. developed an ISD-based method for protein identification, directly from a tissue section, without any further treatment [94]. Indeed, to be implemented, the technique requires only the use of an "ISD-favorable" matrix such as 2,5-DHB or 1,5-DAN as already mentioned. The analysis is then performed as a simple full scan MS acquisition, neither requiring an increase of the laser intensity nor the number of shots on the tissue. The resulting mass spectrum allows the creation of a sequence tag of amino acids and the original protein may be identified thanks to a query in protein databases, using a BLAST search.

Figure 3a shows an ISD mass spectrum recorded on a porcine eye lens with 1,5-DAN as a matrix [94]. Two ions series were detected, leading to two different tags: QPLNPKIIIF EKENFKGHSH ELNGPCPNLK ETGVEQAGSV and WQMDRIRRVS QVPQ, respectively. For the first tag, the interrogation of databases led to the identification of the Beta-Crystallin B2 for three different species (rabbit, bovine, and guinea pig). Nevertheless, some differences exist between the primary sequences of rabbit, bovine, and guinea pig Beta-Crystallins B2. To obtain the N-terminal sequence of the porcine Beta-Crystallin B2, a pMS ${ }^{3}$ sequencing experiment was performed on the same tissue slice. The ion at $\mathrm{m} / \mathrm{z}$ value 1,519.0 was selected and then fragmented. Figure $3 \mathrm{~b}$ depicts the resulting MS/MS spectrum. Almost the entire $b$-ions and $y$-ions series were obtained and the acetylation of the Ala residue was also confirmed, giving access to the full sequence of the $\mathrm{N}$-terminus part of the porcine Beta-Crystallin B2 (51 first amino acids), which is not referenced in databases (SwissProt and TrEMBL). These results open the way towards de novo sequencing, directly from tissue slices. The method was applied for biomarkers discovery $[95,96]$. 


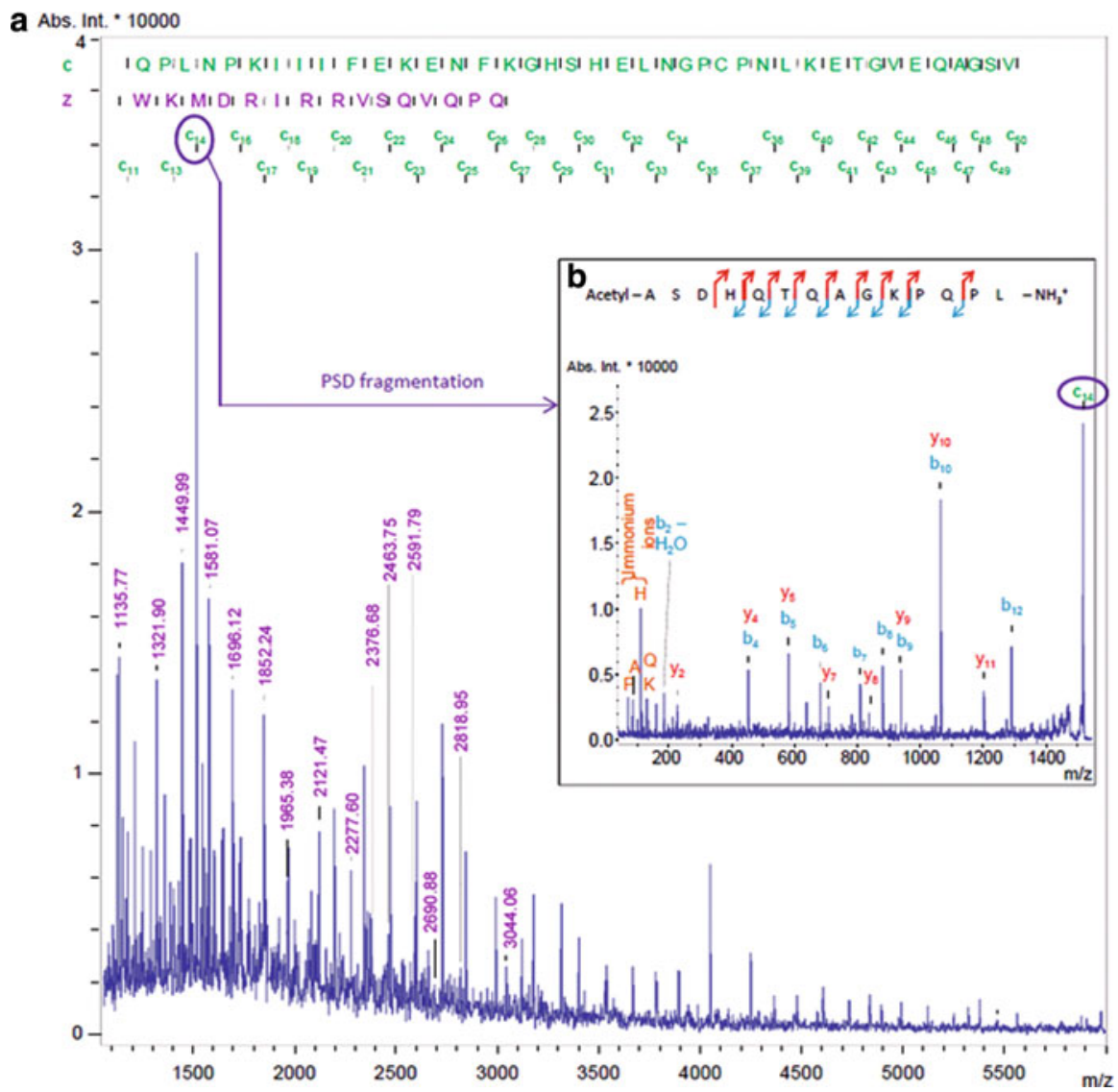

Fig. 3 (a) ISD mass spectrum recorded on a porcine eye lens slice with 1,5-DAN as a matrix. The tag and the masses indicated in green correspond to the $\mathrm{N}$-terminus part of the Beta-Crystallin B2 (c-ions series), the purple ones to the C-terminus part ( $z$-ions series). (b) $\mathrm{T}^{3}$-sequencing mass spectrum obtained by isolating the ion at $m / z 1,519.08$ and by fragmenting it by PSD. Quasi-complete $b$ - and $y$-ions series are observable, leading to the full sequencing of the $\mathrm{N}$-terminus part of the protein

\subsection{MALDI-ISD Imaging}

ISD can also be performed during a tissue imaging experiment, allowing the localization of different fragments to be considered. Figure 4 presents MALDI images recorded on a porcine eye lens slice, with 1,5-DAN as the matrix [94]. Getting the distribution of ISD fragments provides two additional pieces of information. First, it becomes easy to find which fragments are coming from the same protein, as they all exhibit the same localization on the tissue surface. This can help to indicate real consecutive peaks, facilitating the protein sequencing. Second, by overlaying these images, it becomes possible to highlight high intensity pixel(s) and 

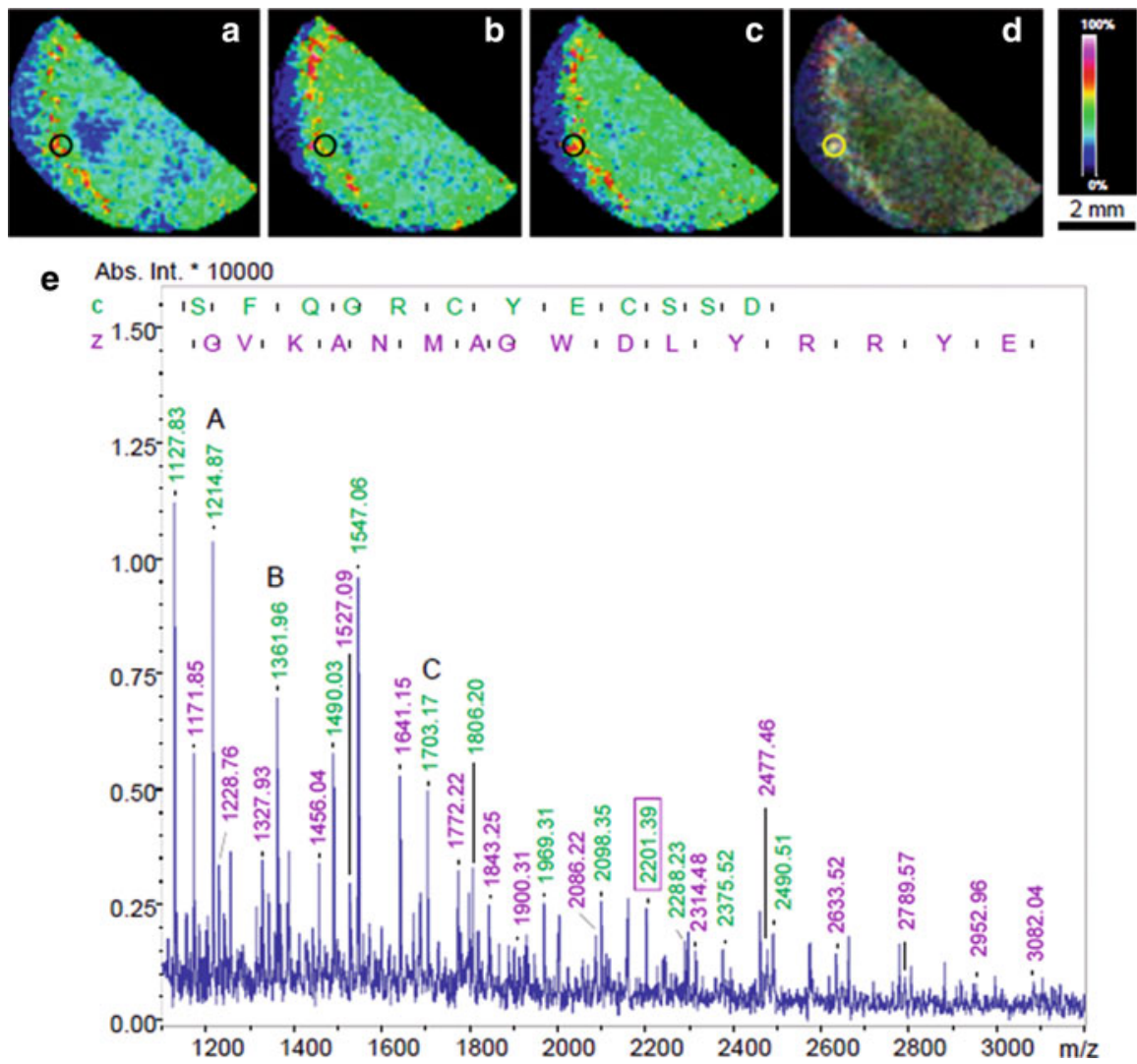

Fig. 4 MALDI images of ISD fragments at (a) $\mathrm{m} / z$ 1,214.9, (b) $\mathrm{m} / \mathrm{z} 1,361.9$, and (c) $\mathrm{m} / \mathrm{z}$ 1,703.2, recorded on a porcine eye lens slice. (d) Overlay of the three previous ion images. The black and the yellow circles indicate the region where the mass spectrum has been extracted (high intensity pixel). (e) ISD mass spectrum extracted from a high intensity pixel. The tag and the masses indicated in green correspond to the N-terminus part of the Gamma-Crystallin B ( $c$-ions series), the purples ones to the $\mathrm{C}$-terminus part (z-ions series). The green purple-framed annotation indicates a peak for which $\mathrm{m} / \mathrm{z}$ value corresponds to both $c$ - and $z$-ion. The ion peaks which images are shown are labeled with letters A, B, C

specifically extract the corresponding mass spectrum. Of course, the more intense the mass spectrum and the more peaks present, the easier the interpretation. In Fig. 4, for example, the three fragments exhibit close localizations, suggesting their common origin, and the overlay of their images (Fig. 4d) shows an intense pixel (yellow-circled) which allows the extraction of the ISD mass spectrum shown in Fig. 4e. Two sequence tags were then established, leading to the identification of Gamma-Crystallin B.

The ISD process is often criticized, and the main denigrations are related to the presumed need for a pure sample, to its lower sensitivity, and to its reduced mass accuracy and resolution. In fact, some of these criticisms are based on false assumptions. 
Concerning the kind of sample that can be analyzed by ISD, it is admitted that a tissue slice is a complex sample and that within one $50-\mu \mathrm{m}$-wide laser shot, several proteins may be desorbed and fragmented. To decrease the number of analyzed proteins, two approaches are considered. The first choice consists of decreasing the size of the laser shot (to 30 or even $20 \mu \mathrm{m}$ ). In this case, the question is: what about the sensitivity of the instrument? Would it be sensitive enough to obtain interpretable ISD mass spectra? How many molecules are present in a 20 - $\mu$ m-wide pixel? And, out of these, how many will fragment? This issue has to be investigated. The second choice for decreasing the number of analyzed proteins is to employ sample depletion methods to remove the high abundance proteins from the tissue slice [97]. Or, rather than depleting the abundant proteins, a variety of identifications can instead be obtained by imaging multiple yet slightly different tissue sections. Thus, in MALDI-ISD imaging, serial sections are still likely to result in detection of the same set of proteins, but the sensitivity of the ISD process to protein ionization efficiencies allows a larger variety of protein identifications to be made from slightly more different tissue sections. MALDI-ISD imaging therefore allows the variety of proteins identified and localized between different tissue sections to mirror more effectively the changes in sample composition. This feature of MALDI-ISD could be used in combination with sample depletion to reveal even more proteins.

Concerning the issue of the mass accuracy, this does not represent a severe problem. Indeed, during the data treatment, it is not the mass of peptides which is measured, but the mass difference between consecutive peaks that is used to obtain an amino acid residue identification. Accurate mass of peptides is more important in the PMF strategy where intact analyte masses are used to query protein databases, but for sequencing a good external calibration of the mass spectrum is sufficient to allow the mass spectrum interpretation. Moreover, to improve the quality of mass accuracy and calibration, it is best to use ISD fragments of a known protein (myoglobin for example) to create a calibration mass list. In this way, data used for calibration and data coming from the sample are recorded under the same conditions and the whole mass range is covered, far better than with a commercial peptides mixture. For mass resolution, as mentioned previously, ISD fragments are formed in the ionization source before the extraction of ions (contrary to PSD), then travel through the flight tube, and with the use of an electrostatic mirror the mass resolution obtained for ISD mass spectra is completely satisfactory.

Progress has recently occurred regarding data treatment for MALDI-ISD imaging. In fact, MALDI-ISD imaging presents a relatively new type of dataset, wherein a protein sequence can be found in each pixel, and this calls for the development of new software and data analysis methods. Zimmerman et al. have created an analytical pipeline suite of software that accomplishes two main goals: automated analysis of MALDI-ISD "top-down" spectra for protein sequencing and automated analysis of MALDI-ISD tissue imaging data [98]. These goals can be combined so that an imaging dataset is first searched by the software to find groups of highly correlated peaks that co-occur and that are likely to correspond to a single protein. Second, the software can then identify the highest quality spectra from the imaging dataset for 
automated de novo sequencing. After a first protein is automatically sequenced or identified, another search is run in the imaging data to find spectral patterns of peaks that are different from that of the first sequenced protein, which are likely to correspond either to a second protein or to a post translationally modified form of the first protein. This semi-automated process is iterated for an exhaustive characterization all of the proteins in the MALDI-ISD imaging dataset. Ion images are subsequently plotted by the software showing the spatial localizations of the identified proteins over the tissue surface. The MALDI-ISD protein sequencing software is used either in combination with imaging datasets, or to sequence proteins from individual MALDI-ISD spectra [98-100].

As applied to individual spectra, the sequencing software uses mass differences calculated from spectral peak lists to perform protein sequencing, and the software is able to overcome interference due to unidentified fragmentation peaks or redundant ion series peaks. In addition, as it was shown that ISD patterns for up to three proteins can exist simultaneously within a single MALDI-ISD spectrum, the software can subtract the already known ISD peak pattern of a known protein out of the peak list. This isolates the remaining peaks, leaving the refined peak list to be passed through the software for identification of a second protein, and by repeating this process a third protein is identified. This analysis process for protein mixtures is advantageous for use with MALDI-ISD tissue imaging data, as MALDI-ISD mass peaks corresponding to more abundant proteins are likely to be already known and can be subtracted from the spectral peak lists to simplify the data analysis for the remaining proteins.

A future possible way to facilitate ISD analysis of complex mixtures is the generation of all possible sequence tags that fit the data within a tolerance window. At present it is difficult for software to interpret ISD mass spectra containing more than two or three proteins because of the large number of peaks present. In the rarer cases where more than three proteins exist in a single ISD spectrum, the idea is to have the software calculate any possible tag and then automatically submit them to query protein databases using a BLAST engine. After this first stage the user should validate (or not) the results and the software could then create an exclusion list for peaks already used, followed by identification of the remaining proteins. This could be seen as a "cleaning" of the ISD mass spectrum. This future direction for automated data treatment would greatly decrease the time needed for mass spectra interpretation and would not necessitate the presence of the user.

\section{Conclusions}

MALDI-ISD seems to have a promising future and will likely have increasing importance in mass spectrometry applications. Top-down sequencing of proteins seems to be the most promising application. Indeed, MALDI-ISD allows a rapid characterization of $\mathrm{N}$-terminal and sometimes $\mathrm{C}$-terminal sequences of a protein and from only a few picomoles of compounds. This technique could be employed 
for the routine quality control of recombinant proteins. Another promising theme resides in MALDI-imaging with ISD and $\mathrm{pMS}^{3}$ performed directly on tissue slices, which could drive the characterization of different proteins and, in the best cases, of pathological biomarkers. In parallel studies, MALDI-ISD has been performed with success on other biopolymers including oligonucleotides and therefore can be generalized as a fast sequencing method.

Acknowledgments The F.R.S.-FNRS (Fonds National de la Recherche Scientifique, Belgium) is acknowledged for postdoctoral fellowships to D.D., T.A.Z., and N.S. The Japanese Society for the Promotion of Science is acknowledged for the postdoctoral fellowship of D.A. The instrumentation was funded by the FNRS, The University of Liege, and the FEDER funds.

\section{References}

1. Rubino FM, Pitton M, Di Fabio D et al (2009) Toward an "omic" physiopathology of reactive chemicals: thirty years of mass spectrometric study of the protein adducts with endogenous and xenobiotic compounds. Mass Spectrom Rev 28:725-784

2. Cox J, Mann M (2011) Quantitative, high-resolution proteomics for data-driven systems biology. Annu Rev Biochem 80:273-299

3. Marchetti-Deschmann M, Allmaier G (2011) Mass spectrometry - one of the pillars of proteomics. J Proteomics 74:915-919

4. Griffiths WJ, Wang Y (2009) Mass spectrometry: from proteomics to metabolomics and lipidomics. Chem Soc Rev 38:1882-1896

5. Cramer R, Gobom J, Nordhoff E (2005) High-throughput proteomics using matrix-assisted laser desorption/ionization mass spectrometry. Expert Rev Proteomics 2:407-420

6. Knochenmuss R (2006) Ion formation mechanisms in UV-MALDI. Analyst 131:966-986

7. Gies AP, Vergne MJ, Orndorff RL et al (2007) MALDI-TOF/TOF CID study of polystyrene fragmentation reactions. Macromolecules 40:7493-7504

8. Konn D, Murrell J, Despeyroux D et al (2005) Comparison of the effects of ionization mechanism, analyte concentration, and ion "cool-times" on the internal energies of peptide ions produced by electrospray and atmospheric pressure matrix-assisted laser desorption ionization. J Am Soc Mass Spectrom 16:743-751

9. Spengler B (1997) Post-source decay analysis in matrix-assisted laser desorption/ionization mass spectrometry of biomolecules. J Mass Spectrom 32:1019-1036

10. Yoon S, Moon J, Kim M (2010) A comparative study of in- and post-source decays of peptide and preformed ions in matrix-assisted laser desorption ionization time-of-flight mass spectrometry: effective temperature and matrix effect. J Am Soc Mass Spectrom 21:1876-1883

11. Kelleher NL (2004) Top-down proteomics. Anal Chem 76:196A-203A

12. Spengler B, Kirsch D, Kaufmann R et al (1991) Metastable decay of peptides and proteins in matrix-assisted laser-desorption mass spectrometry. Rapid Commun Mass Spectrom 5:198-202

13. Karas M, Bahr U, Fournier I et al (2003) The initial-ion velocity as a marker for different desorption-ionization mechanisms in MALDI. Int J Mass Spectrom 226:239-248

14. Syka JEP, Coon JJ, Schroeder MJ et al (2004) Peptide and protein sequence analysis by electron transfer dissociation mass spectrometry. Proc Natl Acad Sci USA 101:9528-9533

15. Demeure K, Gabelica V, De Pauw E (2010) New advances in the understanding of the in-source decay fragmentation of peptides in MALDI-TOF-MS. J Am Soc Mass Spectrom 21:1906-1917 
16. Schulz E, Karas M, Rosu F et al (2006) Influence of the matrix on analyte fragmentation in atmospheric pressure MALDI. J Am Soc Mass Spectrom 17:1005-1013

17. Gabelica V, Schulz E, Karas M (2004) Internal energy build-up in matrix-assisted laser desorption/ionization. J Mass Spectrom 39:579-593

18. Demeure K, Quinton L, Gabelica V et al (2007) Rational selection of the optimum MALDI matrix for top-down proteomics by in-source decay. Anal Chem 79:8678-8685

19. Quinton L, Demeure K, Dobson R et al (2007) New method for characterizing highly disulfide-bridged peptides in complex mixtures: application to toxin identification from crude venoms. J Proteome Res 6:3216-3223

20. Sakakura M, Takayama M (2010) In-source decay and fragmentation characteristics of peptides using 5-aminosalicylic acid as a matrix in matrix-assisted laser desorption/ionization mass spectrometry. J Am Soc Mass Spectrom 21:979-988

21. Smargiasso N, Quinton L, De Pauw E (2011) 2-Aminobenzamide and 2-aminobenzoic acid as new MALDI matrices inducing radical mediated in-source decay of peptides and proteins. J Am Soc Mass Spectrom. doi:10.1007/s13361-011-0307-5

22. Johnson RS, Martin SA, Biemann K et al (1987) Novel fragmentation process of peptides by collision-induced decomposition in a tandem mass spectrometer: differentiation of leucine and isoleucine. Anal Chem 59:2621-2625

23. Bache N, Rand KD, Roepstorff P et al (2008) Gas-phase fragmentation of peptides by MALDI in-source decay with limited amide hydrogen $(1 \mathrm{H} / 2 \mathrm{H})$ scrambling. Anal Chem 80:6431-6435

24. Rand KD, Bache N, Nedertoft MM et al (2011) Spatially resolved protein hydrogen exchange measured by matrix-assisted laser desorption ionization in-source decay. Anal Chem 83:8859-8862

25. Takayama M (2001) N-C $\alpha$ bond cleavage of the peptide backbone via hydrogen abstraction. J Am Soc Mass Spectrom 12:1044-1049

26. Takayama M (2001) In-source decay characteristics of peptides in matrix-assisted laser desorption/ionization time-of-flight mass spectrometry. J Am Soc Mass Spectrom $12: 420-427$

27. Köcher T, Engström Å, Zubarev RA (2005) Fragmentation of peptides in MALDI in-source decay mediated by hydrogen radicals. Anal Chem 77:172-177

28. Calba PJ, Muller JF, Hachimi A et al (1997) Spirooxazines as a molecular probe for the study of matrix-assisted laser desorption/ionization processes. Part I: study of the interaction effect between the molecular probe and the matrix. Rapid Commun Mass Spectrom 11:1602-1611

29. Calba PJ, Muller JF, Inouye M (1998) H-atom transfer following analyte photoionization in matrix-assisted laser desorption/ionization processes. Rapid Commun Mass Spectrom 12:1727-1731

30. Brown RS, Feng J, Reiber DC (1997) Further studies of in-source fragmentation of peptides in matrix-assisted laser desorption-ionization. Int J Mass Spectrom 169-170:1-18

31. Takayama M, Tsugita A (1998) Does in-source decay occur independent of the ionization process in matrix-assisted laser desorption? Int J Mass Spectrom 181:L1-L6

32. Takayama M, Tsugita A (2000) Sequence information of peptides and proteins with in-source decay in matrix assisted laser desorption/ionization-time of flight-mass spectrometry. Electrophoresis 21:1670-1677

33. Hardouin J (2007) Protein sequence information by matrix-assisted laser desorption/ionization in-source decay mass spectrometry. Mass Spectrom Rev 26:672-682

34. Paizs B, Suhai S (2005) Fragmentation pathways of protonated peptides. Mass Spectrom Rev 24:508-548

35. McCormack AL, Somogyi A, Dongre AR et al (1993) Fragmentation of protonated peptides: surface-induced dissociation in conjunction with a quantum mechanical approach. Anal Chem 65:2859-2872

36. Somogyi Á, Wysocki V, Mayer I (1994) The effect of protonation site on bond strengths in simple peptides: application of ab initio and modified neglect of differential overlap bond 
orders and modified neglect of differential overlap energy partitioning. J Am Soc Mass Spectrom 5:704-717

37. Sachon E, Clodic G, Blasco T et al (2009) In-source fragmentation of very labile peptides in matrix-assisted laser desorption/ionization time-of-flight mass spectrometry. Anal Chem 81:8986-8992

38. Asakawa D, Takayama M (2011) C $\alpha$-C bond cleavage of the peptide backbone in MALDI in-source decay using salicylic acid derivative matrices. J Am Soc Mass Spectrom 22:1224-1233

39. Asakawa D, Takayama M (2011) Specific cleavage at peptide backbone $\mathrm{C} \alpha-\mathrm{C}$ and $\mathrm{CO}-\mathrm{N}$ bonds during matrix-assisted laser desorption/ionization in-source decay mass spectrometry with 5-nitrosalicylic acid as the matrix. Rapid Commun Mass Spectrom 25:2379-2383

40. Reiber DC, Brown RS, Weinberger S et al (1998) Unknown peptide sequencing using matrixassisted laser desorption/ionization and in-source decay. Anal Chem 70:1214-1222

41. Brown RS, Lennon JJ (1995) Sequence-specific fragmentation of matrix-assisted laserdesorbed protein/peptide ions. Anal Chem 67:3990-3999

42. Lennon JJ, Walsh KA (1997) Direct sequence analysis of proteins by in-source fragmentation during delayed ion extraction. Protein Sci 6:2446-2453

43. Pfeifer T, Drewello M, Schierhorn A (1999) Using a matrix-assisted laser desorption/ ionization time-of-flight mass spectrometer for combined in-source decay/post-source decay experiments. J Mass Spectrom 34:644-650

44. Suckau D, Resemann A (2003) T3-sequencing: targeted characterization of the N-and C-termini of undigested proteins by mass spectrometry. Anal Chem 75:5817-5824

45. Resemann A, Wunderlich D, Rothbauer U et al (2010) Top-down de novo protein sequencing of a $13.6 \mathrm{kDa}$ camelid single heavy chain antibody by matrix-assisted laser desorption ionization-time-of-flight/time-of-flight mass spectrometry. Anal Chem 82:3283-3292

46. Patterson SD, Katta V (1994) Prompt fragmentation of disulfide-linked peptides during matrix-assisted laser desorption ionization mass spectrometry. Anal Chem 66:3727-3732

47. Schnaible V, Wefing S, Resemann A et al (2002) Screening for disulfide bonds in proteins by MALDI in-source decay and LIFT-TOF/TOF-MS. Anal Chem 74:4980-4988

48. Wefing S, Schnaible V, Hoffmann D (2006) SearchXLinks. A program for the identification of disulfide bonds in proteins from mass spectra. Anal Chem 78:1235-1241

49. Lennon JJ, Walsh KA (1999) Locating and identifying posttranslational modifications by in-source decay during MALDI-TOF mass spectrometry. Protein Sci 8:2487-2493

50. Raska CS, Parker CE, Huang C et al (2002) Pseudo-MS3 in a MALDI orthogonal quadrupole-time of flight mass spectrometer. J Am Soc Mass Spectrom 13:1034-1041

51. van der Wel H, Fisher SZ, West CM (2002) A bifunctional diglycosyltransferase forms the Fuc $\alpha 1,2 \mathrm{Gal} \beta 1,3$-disaccharide on Skp1 in the cytoplasm of Dictyostelium. J Biol Chem 277:46527-46534

52. Hanisch F-G (2011) Top-down sequencing of O-glycoproteins by in-source decay matrix-assisted laser desorption ionization mass spectrometry for glycosylation site analysis. Anal Chem 83:4829-4837

53. Chaurand P, DaGue BB, Ma S et al (2001) Strain-based sequence variations and structure analysis of murine prostate specific spermine binding protein using mass spectrometry. Biochemistry-US 40:9725-9733

54. Harvey DJ, Hunter AP, Bateman RH et al (1999) Relationship between in-source and post-source fragment ions in the matrix-assisted laser desorption (ionization) mass spectra of carbohydrates recorded with reflectron time-of-flight mass spectrometers. Int J Mass Spectrom 188:131-146

55. Harvey DJ, Naven TJP, Küster B et al (1995) Comparison of fragmentation modes for the structural determination of complex oligosaccharides ionized by matrix-assisted laser desorption/ionization mass spectrometry. Rapid Commun Mass Spectrom 9:1556-1561

56. Naven TJP, Harvey DJ, Brown J et al (1997) Fragmentation of complex carbohydrates following ionization by matrix-assisted laser desorption with an instrument fitted with time-lag focusing. Rapid Commun Mass Spectrom 11:1681-1686 
57. Cancilla MT, Penn SG, Carroll JA et al (1996) Coordination of alkali metals to oligosaccharides dictates fragmentation behavior in matrix assisted laser desorption ionization/ Fourier transform mass spectrometry. J Am Chem Soc 118:6736-6745

58. Bashir S, Giannakopulos AE, Derrick PJ et al (2004) Matrix-assisted laser desorption/ ionisation time-of-flight mass spectrometry. A comparison of fragmentation patterns of linear dextran obtained by in-source decay, post-source decay and collision-induced dissociation and the stability of linear and cyclic glucans studied by in-source decay. Eur J Mass Spectrom 10:109-120

59. Yang H, Yu Y, Song F et al (2011) Structural characterization of neutral oligosaccharides by laser-enhanced in-source decay of MALDI-FTICR MS. J Am Soc Mass Spectrom 22:845-855

60. Wuhrer M, Deelder AM (2006) Matrix-assisted laser desorption/ionization in-source decay combined with tandem time-of-flight mass spectrometry of permethylated oligosaccharides: targeted characterization of specific parts of the glycan structure. Rapid Commun Mass Spectrom 20:943-951

61. Yamagaki T, Suzuki H, Tachibana K (2005) In-source and postsource decay in negative-ion matrix-assisted laser desorption/ionization time-of-flight mass spectrometry of neutral oligosaccharides. Anal Chem 77:1701-1707

62. Suzuki N, Khoo K-H, Chen C-M et al (2003) N-glycan structures of pigeon IgG. J Biol Chem 278:46293-46306

63. Yu S-Y, Khoo K-H, Yang Z et al (2008) Glycomic mapping of O- and N-linked glycans from major rat sublingual mucin. Glycoconj J 25:199-212. http://www.ncbi.nlm.nih.gov/pubmed/ 17891558

64. Yu S-Y, Wu S-W, Khoo K-H (2006) Distinctive characteristics of MALDI-Q/TOF and TOF/TOF tandem mass spectrometry for sequencing of permethylated complex type N-glycans. Glycoconj J 23:355-369

65. Terada M, Khoo K-H, Inoue R et al (2005) Characterization of oligosaccharide ligands expressed on SW1116 cells recognized by mannan-binding protein. J Biol Chem 280:10897-10913

66. Klisch K, Jeanrond E, Pang P-C et al (2008) A tetraantennary glycan with bisecting $\mathrm{N}$-acetylglucosamine and the $\mathrm{Sda}$ antigen is the predominant $\mathrm{N}$-glycan on bovine pregnancy-associated glycoproteins. Glycobiology 18:42-52

67. Smargiasso N, De Pauw E (2010) Optimization of matrix conditions for the control of MALDI in-source decay of permethylated glycans. Anal Chem 82:9248-9253

68. Netz DJA, Pohl R, Beck-Sickinger AG et al (2002) Biochemical characterisation and genetic analysis of aureocin A53, a new, atypical bacteriocin from Staphylococcus aureus. J Mol Biol 319:745-756

69. Yoo C, Suckau D, Sauerland V et al (2009) Toward top-down determination of PEGylation site using MALDI in-source decay MS analysis. J Am Soc Mass Spectrom 20:326-333

70. Harris JM, Chess RB (2003) Effect of pegylation on pharmaceuticals. Nat Rev Drug Discov 2:214-221

71. Nordhoff E, Karas M, Cramer R et al (1995) Direct mass spectrometric sequencing of lowpicomole amounts of oligodeoxynucleotides with up to 21 bases by matrix-assisted laser desorption/ionization mass spectrometry. J Mass Spectrom 30:99-112

72. Zhu L, Parr GR, Fitzgerald MC et al (1995) Oligodeoxynucleotide fragmentation in MALDI/TOF mass spectrometry using 355-nm radiation. J Am Chem Soc 117:6048-6056

73. Juhasz P, Roskey MT, Smirnov IP et al (1996) Applications of delayed extraction matrix-assisted laser desorption ionization time-of-flight mass spectrometry to oligonucleotide analysis. Anal Chem 68:941-946

74. McLuckey S, Van Berkel G, Glish G (1992) Tandem mass spectrometry of small, multiply charged oligonucleotides. J Am Soc Mass Spectrom 3:60-70

75. Wu J, McLuckey SA (2004) Gas-phase fragmentation of oligonucleotide ions. Int J Mass Spectrom 237:197-241 
76. Christian N, Reilly J, Mokler V et al (2001) Elucidation of the initial step of oligonucleotide fragmentation in matrix-assisted laser desorption/ionization using modified nucleic acids. J Am Soc Mass Spectrom 12:744-753

77. Chan T, Fung Y, Li Y (2002) A study of fast and metastable dissociations of adenine-thymine binary-base oligonucleotides by using positive-ion MALDI-TOF mass spectrometry. J Am Soc Mass Spectrom 13:1052-1064

78. Wang BH, Hopkins CE, Belenky AB et al (1997) Sequencing of modified oligonucleotides using in-source fragmentation and delayed pulsed ion extraction matrix-assisted laser desorption ionization time-of-flight mass spectrometry. Int J Mass Spectrom 169-170:331-350

79. Chifotides HT, Koomen JM, Kang M et al (2004) Binding of DNA purine sites to dirhodium compounds probed by mass spectrometry. Inorg Chem 43:6177-6187

80. Lemaire R, Menguellet SA, Stauber J et al (2007) Specific MALDI imaging and profiling for biomarker hunting and validation: fragment of the $11 \mathrm{~S}$ proteasome activator complex, Reg alpha fragment, is a new potential ovary cancer biomarker. J Proteome Res 6:4127-4134

81. Kang S, Shim HS, Lee JS et al (2010) Molecular proteomics imaging of tumor interfaces by mass spectrometry. J Proteome Res 9:1157-1164

82. Oppenheimer SR, Mi D, Sanders ME et al (2010) Molecular analysis of tumor margins by MALDI mass spectrometry in renal carcinoma. J Proteome Res 9:2182-2190

83. Pierson J, Norris JL, Aerni H-R et al (2004) Molecular profiling of experimental Parkinson's disease: direct analysis of peptides and proteins on brain tissue sections by MALDI mass spectrometry. J Proteome Res 3:289-295

84. Stauber J, Lemaire R, Franck J et al (2008) MALDI imaging of formalin-fixed paraffinembedded tissues: application to model animals of Parkinson disease for biomarker hunting. J Proteome Res 7:969-978

85. Hsieh Y, Casale R, Fukuda E et al (2006) Matrix-assisted laser desorption/ionization imaging mass spectrometry for direct measurement of clozapine in rat brain tissue. Rapid Commun Mass Spectrom 20:965-972

86. Khatib-Shahidi S, Andersson M, Herman JL et al (2006) Direct molecular analysis of wholebody animal tissue sections by imaging MALDI mass spectrometry. Anal Chem 78:6448-6456

87. Cornett DS, Frappier SL, Caprioli RM (2008) MALDI-FTICR imaging mass spectrometry of drugs and metabolites in tissue. Anal Chem 80:5648-5653

88. Trim PJ, Henson CM, Avery JL et al (2008) Matrix-assisted laser desorption/ionization-ion mobility separation-mass spectrometry imaging of vinblastine in whole body tissue sections. Anal Chem 80:8628-8634

89. Atkinson SJ, Loadman PM, Sutton C et al (2007) Examination of the distribution of the bioreductive drug AQ4N and its active metabolite AQ4 in solid tumours by imaging matrixassisted laser desorption/ionisation mass spectrometry. Rapid Commun Mass Spectrom 21:1271-1276

90. Sugiura Y, Setou M (2010) Imaging mass spectrometry for visualization of drug and endogenous metabolite distribution: toward in situ pharmacometabolomes. J Neuroimmune Pharm 5:31-43

91. Benabdellah F, Touboul D, Brunelle A et al (2009) In situ primary metabolites localization on a rat brain section by chemical mass spectrometry imaging. Anal Chem 81:5557-5560

92. Groseclose MR, Andersson M, Hardesty WM et al (2007) Identification of proteins directly from tissue: in situ tryptic digestions coupled with imaging mass spectrometry. J Mass Spectrom 42:254-262

93. Kaletas BK, van der Wiel IM, Stauber J et al (2009) Sample preparation issues for tissue imaging by imaging MS. Proteomics 9:2622-2633

94. Calligaris D, Villard C, Lafitte D (2011) Advances in top-down proteomics for disease biomarker discovery. J Proteomics 74:920-934

95. Calligari D, Villard C, Terras L et al (2010) MALDI in-source decay of high mass protein isoforms: application to alpha- and beta-tubulin variants. Anal Chem 82:6176-6184 
96. Debois D, Bertrand V, Quinton L et al (2010) MALDI-in source decay applied to mass spectrometry imaging: a new tool for protein identification. Anal Chem 82:4036-4045

97. Bonnel D, Longuespee R, Franck J et al (2011) Multivariate analyses for biomarkers hunting and validation through on-tissue bottom-up or in-source decay in MALDI-MSI: application to prostate cancer. Anal Bioanal Chem 401:149-165

98. Zimmerman TA, Debois D, Mazzucchelli G et al (2011) An analytical pipeline for MALDI in-source decay mass spectrometry imaging. Anal Chem 83:6090-6097

99. Demine R, Walden P (2004) Sequit: software for de novo peptide sequencing by matrix-assisted laser desorption/ionization post-source decay mass spectrometry. Rapid Commun Mass Spectrom 18:907-913

100. Gao J, Tsugita A, Takayama $M$ et al (2002) A programmable fragmentation analysis of proteins by in-source decay in MALDI-TOF mass spectrometry. Anal Chem 74:1449-1457 